\title{
Influencia del orden de matrícula en la elección de grupo, en los resultados académicos y la encuesta de valoración del profesorado
}

\author{
Fernando Cardona $^{\mathrm{a}, \mathrm{b}}$, Juan A. Llorens-Molina ${ }^{\mathrm{c}}$
}

a Instituto de Biomedicina de Valencia. Consejo Superior de Investigaciones Científicas b Departamento de Tecnología de Alimentos. Universitat Politècnica de València. fercarse@ tal.upv.es ${ }^{c}$ E.T.S. Ingeniería Agronómica y Medio Natural. Universitat Politècnica de València

\section{Resumen}

Al inicio del primer curso universitario los alumnos escogen grupo por orden de matrícula, y este orden viene condicionado por la nota obtenida en la Prueba de Acceso a la Universidad. Los profesores observan en general una clara influencia de la nota de este prueba en la capacidad de aprendizaje del alumnado, al menos comparando entre grados con notas de corte considerablemente distintas. En este sentido, la prueba parece ser un buen indicador de esta capacidad de aprendizaje. También se ha observado que, en general, los alumnos con mejores notas en las pruebas de acceso (y preferencia en el orden de matrícula) tienden a escoger el grupo A, y dentro del mismo el subgrupo A1 antes que el A2, independientemente de otros factores como pueden ser el horario o el profesorado que imparte la docencia de cada grupo. Por este motivo, los grupos se completan en el orden alfabético-numérico lógico: primero A1, después el A2, luego el B1, y por último el B2.

En el presente trabajo se analizan las calificaciones por grupos de la Unidad Didáctica 2 (Química Física) de la asignatura Fundamentos Químicos en Ciencia y Tecnología de los Alimentos, de primer curso del Grado en Ciencia y Tecnología de los Alimentos de la Univeristat Politècnica de València. Los resultados demuestran la influencia del orden de matrícula (reflejada como grupo de matrícula) en las calificaciones obtenidas, siendo sustancialmente mejores en el grupo A, incluso en las prácticas de laboratorio, y teniendo en cuenta que es el mismo profesor el que imparte los dos grupos de teoría, y otro profesor el que imparte los dos grupo de prácticas. Además, se ve una clara influencia en la valoración que los alumnos de cada grupo hacen de la asignatura y del profesor, como muestran los resultados de las encuestas de opinión del alumnado, que se realizan después de calificarse la primera convocatoria de la unidad didáctica. 
Palabras clave: Orden de matrícula, capacidad de aprendizaje del alumnado, calificaciones, encuestas de opinión del alumnado.

\section{Introducción}

En el primer curso universitario los estudiantes escogen grupo por orden de matrícula, lo que a su vez es establecido por la nota obtenida en las Prueba de Acceso a la Universidad (PAU). También se ha observado que, en general, los estudiantes que se matriculan primero tienen tendencia a escoger el grupo A, y dentro del mismo el subgrupo A1 antes que el A2, independientemente de otros factores como pueden ser el horario o el profesorado que imparte la docencia de cada grupo. Por este motivo, los grupos se completan en el orden alfabético-numérico lógico: primero A1, después A2, luego el B1, y por último el B2, en el caso de que haya cuatro subgrupos. Por otro lado, el profesorado observa una clara influencia de la nota del PAU en la capacidad de aprendizaje, al menos comparando entre grados con notas de corte considerablemente distintas. En este sentido, la prueba del PAU parece ser un buen indicador de las capacidades de los estudiantes.

Se ha observado en algunos caso la influencia del grupo de matrícula en los resultados académicos a favor de los grupos ordenado por orden alfabético (Campo-Cabal, 2012), aunque la diferencia no resultara estadísticamente significativa con los tests aplicados, o bien fuera debida a las diferencias entre el profesorado o los métodos de evaluación.

En lo que respecta a la evaluación de los docentes universitarios, el artículo 108 de los Estatutos de la Universitat Politècnica de València (UPV) establece que el Consejo de Gobierno establecerá los criterios de evaluación de la actividad y dedicación docente, de gestión e investigadora y la contribución al desarrollo científico, tecnológico o artístico del personal docente e investigador, que serán relevantes para determinar su eficiencia en el desarrollo de su actividad. A este respecto en el Manual de Evaluación de la Actividad Docente del Profesorado (Índice de Actividad Docente) se regula la actual encuesta de opinión del alumnado, siendo competencia del Instituto de Ciencias de la Educación la lectura de las encuestas y posterior tratamiento de datos, así como la elaboración de los informes personales, los de cada Estructura Responsable de Título, Departamento y Rectorado. De este artículo y su aplicación se desprende que la principal, aunque no la única, herramienta de evaluación del profesorado universitario de la UPV son las encuestas de opinión del alumnado.

La objetividad y validez de estas encuestas ha sido cuestionada en repetidas cocasiones (Silva-Montes, 2009), incluso dentro de la UPV (Verdeguer et al, 2013), o bien se ha concluido que solo es válida para evaluar algunos aspectos de la docencia (Zambrano et al, 
2015). También se ha postulado que la evaluación del profesorado depende del rendimiento académico de los estudiantes (La Serna Studzinski, 2014), del interés previo por el tema de estudio (Marsh, 1987), o las calificaciones esperadas o recibidas, por lo que se plantea en numerosas ocasiones si deberían realizarse antes de que el alumno sea evaluado (Aleamoni y Hexner, 1980; Aparicio et al, 1982; De Salvador, 1996; revisado en Marsh, 2007).

En este trabajo se estudia la influencia del grupo de matrícula en el rendimiento académico de una asignatura de prier curso, así como la influencia de las calificaciones obtenidas en la evaluación del profesorado. Los resultados obtenidos apuntan a que la nota obtenida en el PAU, reflejada en el grupo de matrícula, afecta a las capacidades académicas de los estudiantes, y ésta a su vez a la opinión que tienen del profesorado y de la asignatura.

\section{Contexto}

El presente trabajo analiza la influencia del grupo en los resultados académicos y en la encuesta de opinión del alumnado, tanto en la parte de teoría (TA, impartida por el mismo profesor en ambos grupos) como en prácticas de laboratorio (PL, impartida en los cuatro grupos por la misma profesora). El contexto es la unidad didáctica 2 de la asignatura Fundamentos Químicos para la Ciencia y Tecnología de los Alimentos, del primer curso del correspondiente grado.

Para evaluar los resultados académicos se utilizaron las calificaciones de los alumnos de teoría y prácticas. Para la opinión de los alumnos sobre el profesor se utilizaron las evaluaciones del profesor de teoría, ya que eran las únicas disponibles.

\section{Resultados y discusión}

\subsection{Influencia del grupo de matrícula en los resultados académicos}

El análisis de los resultados académicos por grupos, muestra que las calificaciones obtenidas por el grupo A son significativamente más altas que las del B (Fig. 1), tanto en el caso de TA (Fig. 1a, t-student $\mathrm{p}=0,038$ ) como para la parte de PL (Fig. 1b, t-student $\mathrm{p}=$ 0,0048). Cabe destacar que en ambos casos el profesor es el mismo para los dos grupo, y distinto para TA y PL, por lo que queda descartada la influencia del profesor en los resultados.

Al separar las calificaciones obtenidas en TA en el examen de teoría (EXTA) y el de problemas (EXPR), se observa que la diferencia es debida principalmente a las 
calificaciones obtenidas en la parte de problemas (Fig. 1a). Separar las notas por tramos (5$6,7-8,9-10)$, no permite atribuir la diferencia a ningún tramo concreto, si no que la disminución ocurre significativamente en todos ellos (datos no mostrados).

En el caso de las PL, la diferencia se debe a su realización en el laboratorio (PL, t-student p $=0,035)$, y no al examen de prácticas (EXPL, t-student $\mathrm{p}=0,129)$ (Fig. 1b). Sin embargo, no se encontraron diferencias significativas para los diferentes subgrupos de prácticas (A1 respecto $\mathrm{A} 2$, $\mathrm{B} 1$ respecto $\mathrm{B} 2$, datos no mostrados).

a)

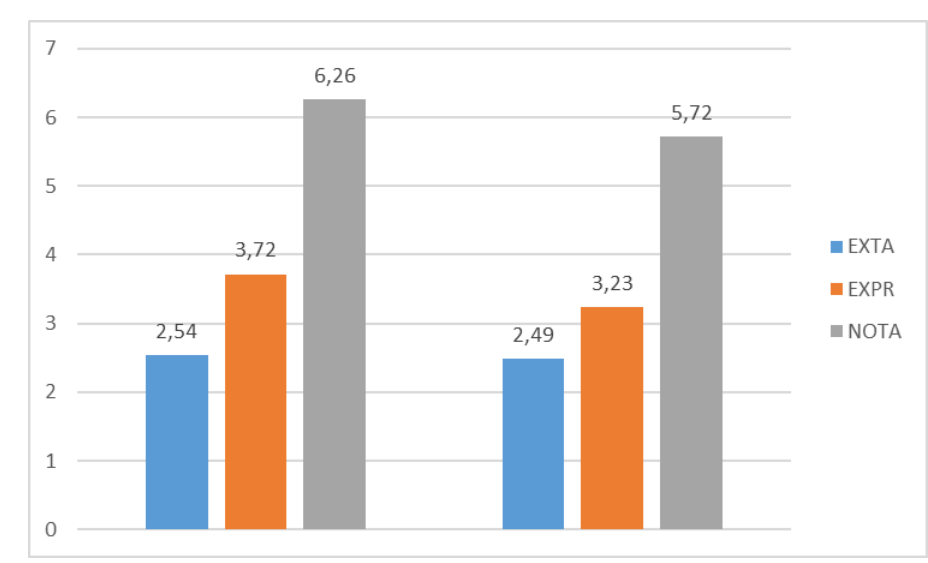

b)

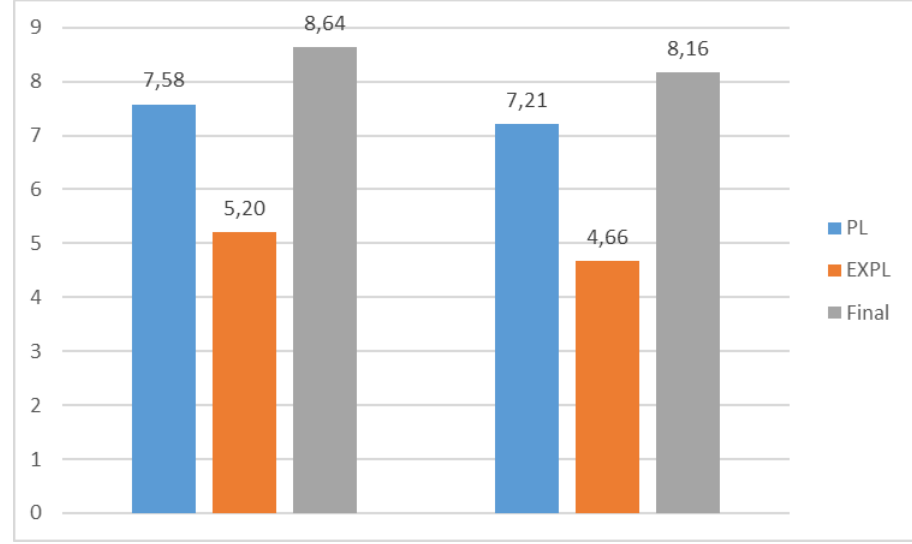

Grupo A

Grupo B

Fig. 1. Comparativa entre grupos de las resultados obtenidos en teoría (a) y prácticas (b). Se muestra sobre la barra del histogrma la media de las calificaciones. Fuente: elaboración propia. 


\subsection{Influencia de los resultados académicos en los resultados de opinión del alumnado}

Al analizar los resultados de las encuestas de opinión del alumnado por grupos (es como se le proporcionan al profesor), se observa también que los resultados son diferentes según el grupo considerado, siendo bastante peor la opinión del grupo que ha obtenido peores calificaciones (Fig. 2). Separando los resultados de la encuesta en los diferentes apartados en los que se proporciona, se observa que las diferencias son comunes a todos, y por lo tanto no dependen de la parte que se considere.

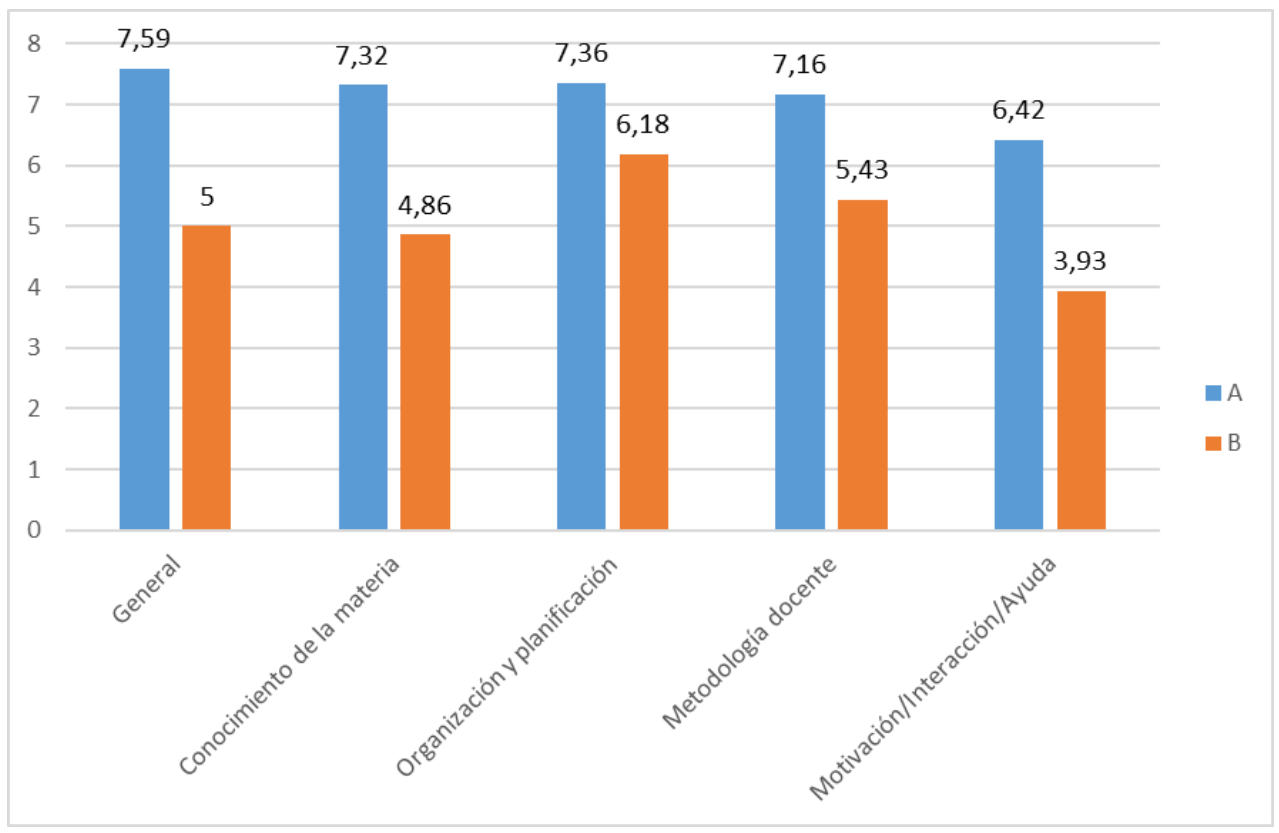

Fig. 2. Comparativa entre grupos de los resultados de las encuestas de opinión del alumnado para el profesor de teoría. Se muestra sobre la barra del histogrma la media de las calificaciones. Fuente: elaboración propia.

\section{Conclusiones}

4.1. El grupo de matrícula de los estudiantes afecta a los estudiantes de esta asignatura en este curso académico, siendo mejores los resultados del grupo A que los del grupo B.

4.2. Los resultados académicos obtenidos en la asignatura afectan a la opinión que los estudiantes tienen sobre el profesor y la asignatura, al menos en esta asignatura y curso académico.

4.3. Es necesario un seguimiento en cursos posteriores con diferentes promociones de alumnos para corroborar los resultados obtenidos. En caso de confirmarse, sería necesario replantearse el protocolo de matrícula y de evaluación del profesorado. 


\section{Referencias}

Aleamoni, L.M. y Hexner, P.Z. (1980). A review of the research on student evaluation and a report on the effect of different sets of instructions on student course and instructor evaluation. Instructional Science, 9 (1), 67-84.

Aparicio, J.J., Tejedor, F.J. y Sanmartín, R. (1982). La enseñanza universitaria vista por sus alumnos: Un estudio para la evaluación de los cursos de la enseñanza superior. Madrid: ICE/ Universidad Autónoma de Madrid.

Campo-Cabal, G. (2012). Evaluación de un sistema de evaluación del aprendizaje en psiquiatría. Revista Colombiana de Psiquiatría, 41(1), 87-103

De Salvador, X. (1996). Sobre la evaluación de la actividad docente del profesorado universitario: ¿Está mediatizada la valoración de los alumnos por las calificaciones? Revista Española de Pedagogía, 203, 107-128.

Encuestas UPV: https://www.upv.es/contenidos/ICEP/info/944077normalc.html

Karlos La Serna Studzinski, K., Becerra Marsano, A.M.,Beltrán Barco, A. y Zhang, H (2014). La relación de las encuestas de evaluación docente con el rendimiento académico: La evidencia empírica en la Universidad del Pacífico. Revista Iberoamericana de Evaluación Educativa, 7(2), 105-115.

Marsh, H.W. (2007). Students' Evaluations of University Teaching: Dimensionality, Reliability, Validity, Potential Biases and Usefulness. En R.P. Perry and J.C. Smart (eds.), The Scholarship of Teaching and Learning in Higher Education: An Evidence- Based Perspective, (pp.319-383). Netherlands: Springer.

Silva-Montes, .C, (2009). Las Encuestas de Opinión en la Universidad Autónoma de Ciudad Juárez: ¿Un Caso de Exclusión del Profesorado?. Archivos Analíticos de Políticas Educativas. 17(24), 134.

Verdeguer, M., Reig, C., Peiró, R., Guijarro, E. (2013). ¿SON LAS ENCUESTAS DE EVALUACIÓN DEL PROFESORADO VÁLIDAS PARA LAS NUEVAS METODOLOGÍAS DE ENSENAANZA? Riunet (repositorio UPV)

Zambrano R., Gil, N., Lopera, E., Carrasco, N. (2015). Validez y confiabilidad de un instrumento de evaluación de estrategias docentes en la práctica de la ensenanza universitaria. Magister 27, 2636. 